\title{
Eukaryotic Endoplasmic Reticulum Stress-Sensing Mechanisms
}

\author{
Lillian E. Duffee ${ }^{1}$, Jon L. Boatwright ${ }^{1}$, Faris H. Pacha ${ }^{1,2}$, James M. Shockley ${ }^{1}$, \\ Karolina M. Paje rowska-Mukhtar ${ }^{1}$, M. Shahid Mukhtar ${ }^{1, *}$ \\ ${ }^{1}$ Department of Biology, University of Alabama at Birmingham, Birmingham, AL 35294, USA \\ ${ }^{2}$ Birmin gham-Southern College, Birmingham, AL 35254, USA
}

\begin{abstract}
The endoplasmic reticulum (ER) is responsible for proper fold ing of secretory and membrane-bound proteins as well as the degradation of improperly folded proteins. Fluctuations in protein fold ing demand exceed ing fold ing capacity result from events such as cellular stress and mutations affecting protein folding. Detrimental accumulation of unfolded proteins within the ER is alleviated through activation of evolutionarily conserved, intracellular signaling proteins by the unfolded protein response (UPR). Binding of membrane-bound signaling proteins by inactive, ER-resident chaperones typically results in suppression of UPR. However, upon nascent protein accumulation, chaperone recruitment allows for activation of stress relieving pathways. UPR, classically studied in budding yeast and later in metazoan and plant cells, relies almost exclusively on the signal proteins, known as Ire1p. Homologs for the metazoan UPR sensors include Ire $1 \alpha$ and Ire $1 \beta$ as well as two addit ional signal proteins, PERK and ATF6. Plant UPR branches identified to date include IRE1a, IRE1b, bZIP17 and bZIP28. In this review, we present the first comprehensive view of both conserved and differing aspects of UPR across kingdoms, with special emphasis on some unique features of recently discovered plant UPR pathways.
\end{abstract}

Keywords Endoplasmic Reticulum Stress, Unfolded Protein Response, IRE1, PERK, ATF6

\section{Introduction}

The fold ing of nascent proteins is essential to reaching their correct three-dimensional conformation and eventual proper function. In the eukaryotic system, the endoplasmic reticulum (ER) is responsible for housing the necessary chaperones that facilitate proper folding[1]. Throughout a cell's life, demand for folding fluctuates. Periodically, the ER acquires an excess of unfolded proteins and fails to meet the folding requirements, resulting in ER stress [2]. Unfolded protein accumu lation and ER stress also occur in a nu mber of medical conditions. The unfolded protein response (UPR) is a cellular response that resolves ER stress through translational inhibition, increased expression of genes encoding ER-resident chaperones, ER expansion and increased activation of ER-Associated Degradation (ERAD) elements. However, if the cell cannot regain homeostasis through these activities, apoptotic pathways are activated.

Initiation of stress mediation begins with activation of membrane-bound ER signaling components[2]. During an unstressed state, their cytosolic domains are occupied by

* Corresponding author:

smukhtar@uab.edu (M. Shahid Mukhtar)

Published online at http://journal.sapub.org/als

Copyright (C) 2012 Scientific \& Academic Publishing. All Rights Reserved inactive ER chaperones, preventing activation of stress pathways[2]. Upon nascent protein accumulation, chaperones dissociate from the ER-resident proteins, triggering activation of the UPR pathways. These me mbrane-bound proteins are each uniquely responsible for initializing signal transduction through pathways consisting of phosphorylation events and dimerization, dissociation from the ER membrane followed by proteolytic cleavage, or even phosphorylation, dimerization and a subsequent endoribonuclease domain activation resulting in an atypical, cytoplasmic splicing event. These pathways ultimately result in recovering ER homeostasis; however, if the ER stress is too profound and irreversible, the cell will initiate the apoptotic program[3].

Glycosylation and deglycosylation cycles give misfolded proteins a way to rema in in the ER to undergo proper fold ing. If proper folding does not occur, ER-associated degradation (ERAD) pathway provides a mechanism for removing such proteins. Terminal mannose residues are removed by ER $\alpha$-mannosidase I, subsequently bound to ER degradation enhancing $\alpha$-mannosidase like proteins (EDEM) and transported to the cytosol, where the unfolded proteins are degraded by the ubiquitin-proteasome pathway[1, 4, 5].

In addition to glycosylation and deglycosylation cycles, correct folding is aided by chaperones, which bind to unfolded proteins in ways that increase both the likelihood of 
correct folding and the length of time these proteins reside in the ER. ER luminal binding protein (BiP) is a member of the critical Hsp70 family of molecular chaperones. In addition to serving as a folding chaperone, BiP binds to exposed hydrophobic regions of unfolded proteins prompting dissociation from and activation of UPR receptors. Additionally, BiP serves as an ER stress regulator by buffering calcium levels and preventing the activation of pro-apoptosis signals by binding to caspase proteins $[1,4,5]$.

Characterization of the UPR signaling elements began in baker's yeast, Saccharomyces cerevisiae, almost two decades ago. The yeast genome encodes a protein kinase, Irelp (inositol-requiring enzyme 1) that is responsible for UPR signal transduction. The mammalian suite of UPR components comprises: Ire $1 \alpha / \operatorname{Ire} 1 \beta$, homo logs of the yeast Ire1p; ATF6, activated transcription factor 6; and PERK, a PKR-like ER-resident eIF2 $\alpha$ kinase[2]. Lastly, known regulators of plant UPR include IRE1a/IRE1b, constituting IRE1 homologs, and bZIP17/bZIP28 transcription factors, equivalents of the ATF6 pathway in mammals. In addition to possessing additional UPR signaling pathways, the existence of multiple copies of sensors in higher organisms has been suggested to enable more sophisticated and selective function, such as the presence of Ire $1 \alpha$ and Ire $1 \beta$ proteins in mammals compared to only one copy of IRE1 in C. elegans and D. melanogaster[6].

Furthermore, the expansion of IRE1a and IRE1b, as well as the presence of the bZIP17/bZIP28 proteins and a GCN2 (General control non-derepressible-2) homolog in plants (discussed below), appears to allow for expanded detection of both abiotic and biotic stress through a more selective stress sensing by each protein[7-10].

Current research also suggests that UPR signal transducers affect a greater number of targets than originally thought. Specifically, the ability of IRE1 $\alpha$ to bind to and cleave various mRNAs has been recently explored. Using a combination of cleavage assays and exon array analysis, 13 novel mRNAs were identified as candidate IRE1 $\alpha$ targets[11]. This finding also supports the interpretation of a weak immune-related phenotype observed in the absence of functional bZIP60 as indicative of the existence of other, currently unidentified IRE1 targets in Arabidopsis[10].

Of increasing interest is UPR's role in pathophysiology. UPR activation has been observed in a multitude of diseases and disorders including cancer, diabetes and neurodegenerative diseases. Proliferation of tumor cells leads to oxidative stress and hypoxia that is alleviated via the IRE1 pathway[2]. UPR also functions in glucose homeostasis during energy deprivation and diabetic states, acting to conserve cell energy and improve cell survival. Without the UPR, pancreatic $\beta$-cells would not survive oscillating blood glucose levels[2]. Additionally, dysfunction of the ER can be caused by expanding polyglutamine repeats or neurodegenerative diseases and results in accumulation of atypical proteins, potentially leading to Alzheimer and Parkinson disease[12]. Yeast irel mutant is sensitive to ER stress, while Arabidopsis plants lacking functional IRE1 genes display diminished capacity to trigger effective defense responses to a broad range of pathogens[10].

In this review we elucidate the specific interactions involved in regulation of ER stress via the UPR among eukaryotes and the varying levels of conservation among the systems. We review the current state of knowledge on UPR in yeast and other fungi, and provide insights into the more complex UPR sensing mechanisms found in mammals and, recently, also in plants. Starting with UPR activation, we will detail the specific molecular processes necessary for ER homeostasis and cell survival as well as reveal gaps in current knowledge.

\section{UPR in Yeast and Other Fungi}

UPR in Saccharomyces cerevisiae is now known to consist of a comparatively simple set of pathways, in which only one protein kinase, namely Ire 1p, med iates UPR.

\subsection{Ire1p}

The initial trigger of UPR in yeast, Irelp is a transmembrane kinase and endonuclease protein activated by ligand-triggered dimerization and subsequent trans-autophosphorylation. An accumulation of unfolded proteins is sensed by its core lu minal do ma in (cLD) leading to activation. Once activated, Ire $1 \mathrm{p}$ demonstrates endoribonuclease activity, cleaving HAC1 mRNA into a form, which translates into a basic leucine zipper (bZIP) transcription factor protein known as Hac 1p[13].

Although it is well understood that dissociation from BiP plays a role in Ire 1 activation, the exact mechanism of Ire 1 regulation has been unclear. A recently published study[14] suggests that, rather than a simple ON/OFF switch, $\mathrm{BiP}$ is more of a buffer regulating the concentrations of uninhibited Ire1, which can be activated. This conclusion was reached in part by findings that Ire1 regulation was not completely lost in yeast irel mutant strains unable to bind to BiP. Given this, a two-step model was proposed, in which first BiP dissociation from Ire 1p a llows for Ire 1p oligo merization, and second, Ire $1 \mathrm{p}$ binding to unfolded proteins leads to activation[14]. By sequestering inactive Ire 1 molecules, BiP provides a threshold ensuring access to high concentrations of Ire $1 p$ only during severe stress. This conclusion was further supported by findings of increased clustering - but not activation - among mutant irel strains unable to bind to BiP. Moreover, computational modeling demonstrated increased sensitivity to triggering an UPR in the absence of BiP binding[14].

As expected, irel or hacl-knockout yeast cells demonstrate increased sensitivity to ER stress. Although yeast cells do not possess PERK proteins, they have been found to contain a functional Gen2p. An evolutionary ancestor to mammalian GCN2 and PERK, Gcn2p causes translation attenuation upon starvation stress by 
phosphorylating the eukaryotic initiation factor $2 \alpha$ (eIF $2 \alpha$ ) [6]. Moreover, ER chaperones and ERAD components are simultaneously transcriptionally induced via the Irelp-Hac 1p pathway owing to the absence of ATF6 in yeast[6].

Despite multiple universal aspects of fungal UPR, variance has been found to exist between the changes made to HAC1 mRNA in yeast and other fungi. In S. cerevisiae, a 252 nucleotide intron is spliced fro $\mathrm{mHAC} 1 \mathrm{mRNA}$ resulting in replacement of the carboxy-terminal amino acids in unspliced Haclp (termed Hac lup) with a new 19 amino-acid segment (Haclip)[15]. Other types of fungi, such as Trichoderma reesei and Aspergillus nidulans, exhibit removal of a much smaller, 20-nucleotide intron from their HAC1 homologs. Furthermore, the 5' untranslated regions (UTR) of these fungi are truncated and a unique type of transcriptional down-regulation has been observed[16].

Variance has also been observed between the mechanisms of the HAC 1 translational block in fungi. In $S$. cerevisiae, a translational block acts on HAC1 due to base pairing between the 5' UTR and the unconventional intron; upon excision of this intron, the translational block is removed, allowing for increased production of Haclp. In contrast, the translationalblock on HAC1 in Aspergillus niger is mediated by a GC-rich inverted repeat; upon activation the 5' UTR is truncated, removing part of this repeat and allowing for translation[16].
HAC1 transcription is also unique in that it has been shown to be autoregulated, as Haclp itself binds to an Unfolded Protein Response Element (UPRE), a cis-regulatory motif present in $H A C l$ promoter, to increase its own transcription. The regulation of $\mathrm{Hac} 1 \mathrm{p}$ by a conditional splicing event followed by binding to its own promoter is advantageous as it permits translation only under conditions under which it is needed and allows for a quick increase in Haclp levels to sufficiently relieve ER stress[17].

The fungal pathogen, Aspergillus fumigatus, has also been shown to employ the same IreA-HacA pathway as other fungi, in order to trigger UPR. However, outside of this cellu lar state, Ire $1 \mathrm{p}$ in A. fumigatus has been shown to play a role in multiple adaptive roles, such as thermotolerance, growth under hypoxia, membrane composition and nutritional versatility [18].

A recent report further supported a model of Ire1p activation, in which unfolded proteins serve as ligands facilitating Ire1 activation by oligomerization[4]. The core ER luminal domain (cLD) of Irelp was found to bind to peptides by two interfaces, the first of which facilitates dimer formation and the second promotes further oligomerization. cLD was also found to bind to basic residues, with a particularly high affin ity to arginine[4].

\section{Mammalian UPR}

\subsection{IRE1}

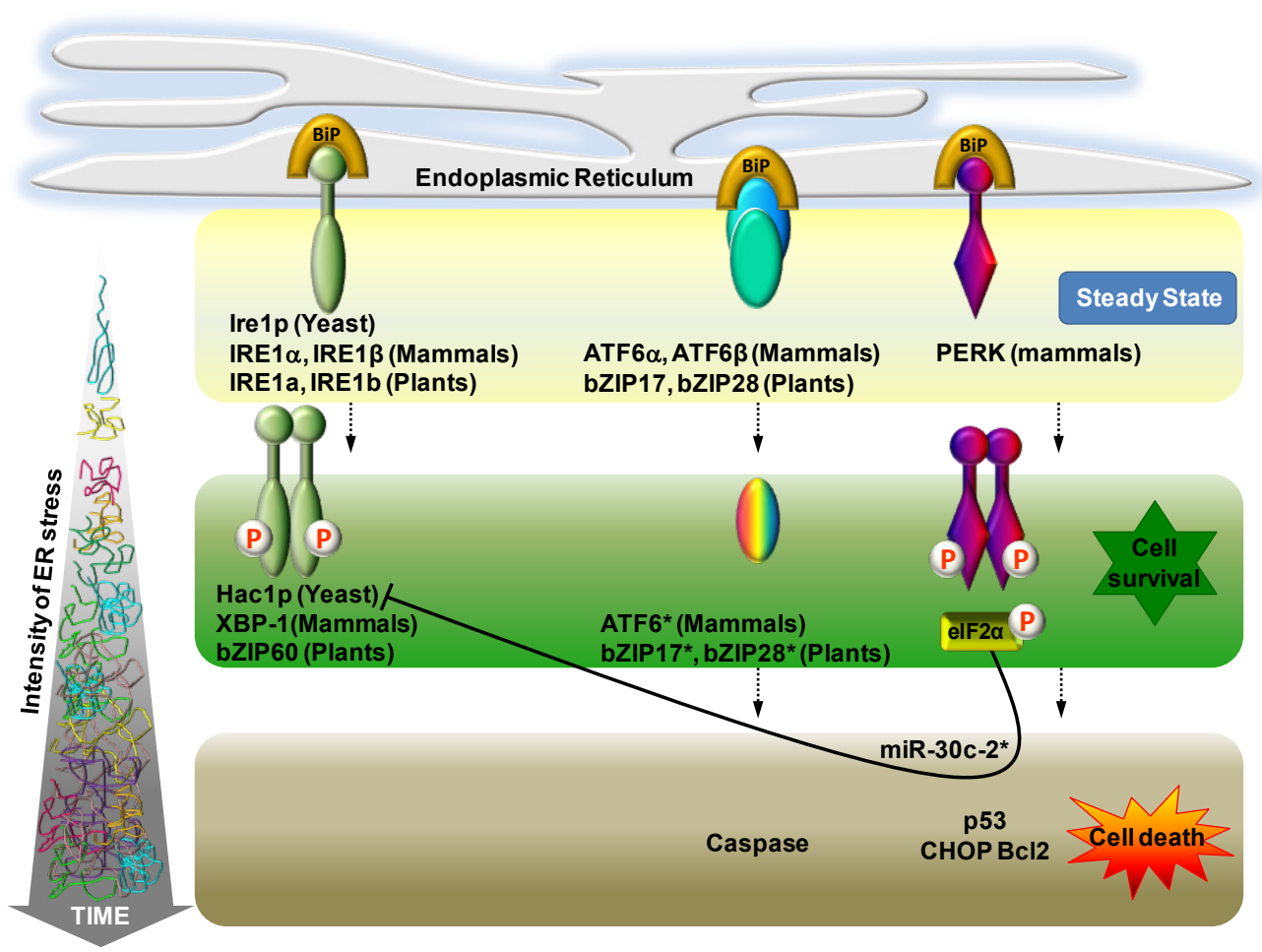

Figure 1. Eukaryotic Unfolded Protein Response pathways. Dist inct UPR pathways in yeast, mammals and plants are demonstrated. In yeast, UPR relies almost exclusively on the signal proteins, known as Irel p. ER stress in mammals ismediated by three ER-resident transmembrane proteins (IRE1 $\alpha /$ IRE1 $\beta$, AT F6 $\alpha / A T F 6 \beta$ andPERK). Plant UPR depends upon IRE1 a/IRE1b and bZIP17/bZIP28). Downst ream signaling in steady state, cell survival and cell death stages are presented. Intensity of the ER stress in terms of malfolded proteins over the course of time is shown. Circled "P" denotes for trans-aut ophosphorylation in its cytosolic kinase domain, while “*” symbolizesthe act ivated form of mammalian AT F6 or plant bZIP proteins 
The most evolutionarily conserved component of UPR, IRE1 exhibits both endoribonuclease and kinase activities across species. As seen in other organisms, of the two IRE1 homologs, IRE1 $\alpha$ (IRE1a) and IRE1 $\beta$ (IRE1b), IRE1 $\alpha$ plays a more central role than IRE1 $\beta$. Moreover, in mammals, IRE $1 \alpha$ is expressed in a diversity of tissues while IRE $1 \beta$ is only found in intestinal epithelia[19-21].

In resting cells, the IRE1 is bound to luminal binding protein (BiP), also referred to as GRP78[4]. Upon interaction with unfolded proteins, BiP dissociates from these receptors. Malfolded peptides accumulating in the ER lumen lead to ER stress that, in turn, causes dissociation of BiP from the luminal IRE1 domains. Specifically, the exposed hydrophobic residues stimulate the ATPase domain of BiP, resulting in an ADP-bound form with a high affinity for hydrophobic regions. BiP is then dissociated from unfolded/misfolded proteins by nucleotide exchange factors (NEFs) such as BiP-Associated Protein (BAP). IRE1, once freed from $\mathrm{BiP}$, undergoes dimerization and transautophosphorylation. Activated IRE1 catalyses the exc ision of a 26 nucleotides long, unconventional intron from XBP-1 (X-Box Binding Protein) mRNA, in a manner mechanistically similar to pre-tRNA splicing. Removal of this intron causes a frame shift in the XBP- 1 coding sequence resulting in the translation of a 371 amino acid, $54 \mathrm{kDa}$, $\mathrm{XBP}-1 \mathrm{~s}$ isoform rather than the 261 amino acid, $33 \mathrm{kDa}$, $\mathrm{XBP}-1 \mathrm{u}$ is oform[22].

The resulting XBP-1s dimerizes and, in conjunction with co-regulators, controls expression of various chaperones and degradation-related proteins. XBP1-s also upregulates $\mathrm{p} 58$, which has been shown to negatively regulate PERK activity and is an example of the interconnected nature of UPR sensor pathways and overall cellular signaling seen in mammals $[6,20,21]$.

Activation of IREla can also engage "alarm" genes by recruiting the adaptor protein TNFR-associated factor 2 (TRAF2). This results in the activation of the apoptosis signal-regulating kinase 1 (ASK1; also known as MAP3K5) pathway and its downstream target c-JUN N-terminal kinase JNK28. In addition, IRE1a also engages alarm pathways such as p38, extracellu lar signal-regulated kinase (ERK) and nuclear factor $\kappa \mathrm{B}(\mathrm{NF} \kappa \mathrm{B})$ through the binding of distinct adaptor proteins [23].

Despite a high level of Ire 1 conservation between species, the cLD of human Ire $1 \alpha$, unlike yeast, was found to have a groove too narrow for peptide binding. One interpretation of this finding is that this represents a closed confirmation of the protein and that upon peptide binding, an open confirmation similar in shape to yeast Irelp is triggered[24]. Multiple proteins have been shown to play a role in IRE1 regulation, including tyrosine phosphatase 1B (PTP-1B), ASK1-interacting protein 1 (AIP1) and members of the Bcl-2 protein family[25-27]. A lthough no direct interaction between PTP-1B and IRE1 has been found, in the absence of PTP-1B decreased IRE1 activity has been shown in the forms of decreased JNK28 activation, XBP- 1 splicing and EDEM transcription[28]. AIP1 has also been shown to interact with both TRAF2 and IRE1 $\alpha$; it is currently thought that AIP1 facilitates IRE1 $\alpha$ dimerization and IRE1 $\alpha$-TRAF2 complex formation, leading to ASK1-JNK signaling activation[25]. Furthermore, IREla has been shown to directly bind to Bax and Bak, two pro-apoptotic members of the Bcl-2 family. It is also thought that Bax inh ibitor 1 (BI-1), an anti-apoptotic protein, increases cell survival by down-regulating IRE1 $\alpha$, through inhibition of its endoribonuclease activity, as well as down-regulating ATF6 (described below)[25-27]. Lastly, members of the heat shock protein (HSP) family have been shown to exhibit IRE1 $\alpha$ regulatory functions. For examp le, HSP 90 has been shown to bind to the cytosolic domain of IRE1 $\alpha$ in such a way that it becomes insulated from degradation by the proteasome. Additionally, HSP72, when bound to the cytosolic doma in of IRE1 $\alpha$, increases the endoribonuclease activity of $\operatorname{IRE} 1 \alpha[23]$.

\subsection{ATF6}

A second ER transmembrane protein, activating transcription factor 6 (ATF6) is a bZIP-like protein that upregulates pro-survival transcription signals to alleviate ER stress by acting as a mediator in the process. Two homologs of ATF 6 have been identified in mammals: ATF6 $\alpha$ and ATF6 3 [29].

As shown in the IRE1 pathway, ER stress leads to dissociation of BiP from the three UPR signal inducers, PERK, ATF6 and IRE1, a llowing for their activation. ATF6, unlike PERK and IRE1, uniquely translocates to the Golgi apparatus where it is modified into its active form by two proteolytic cleavage events. Although not certain, it is thought that Golgi localization sequences, GLS1 and GLS2, take effect with the dissociation from $\mathrm{BiP}$, mediating translocation[29-31]. Also unique among the UPR sensors, ATF6 is not activated by phosphorylation but by regulated intramembrane proteolysis (RIP) in the Golgi system. A first cleavage on the luminal domain by serine protease site-1 protease (S1P) triggers a second cleavage by metalloprotease site-2 protease (S2P). This releases the transcriptional domain for import into the nucleus, where it induces transcription of genes with ATF/cAMP response elements (CREs) and ER stress elements (ERSEs)[6, 29-32].

In conjunction with bZIP transcription factors and co-regulators, ATF6 upregulates chaperone activity and unfolded protein degradation. ATF6 likewise upregulates transcription of $\mathrm{BiP}$, protein disulfide isomerase (PDI) and ER degradation-enhancing alpha-mannosidase like protein 1 (EDEM 1) and induces expression of XBP-1 via activation of the ER stress element (ERSE) in their promoters. XBP-1 constitutes an important intersection between UPR sensors given that after induction by ATF6, XBP-1 is processed by IRE1 $\alpha$ allowing for further induction of chaperones, as well as p58, a negative regulator of PERK[6, 30, 31]. ATF6 exhibits anti-apoptotic effects through induction of a calcineurin regulator, which ultimately leads to the sequestering of $\mathrm{Bc} 12$, a pro-apoptotic protein.

The mechanism by which ATF6 is deactivated is not 
currently known. It has been suggested that an unspliced form of XBP-1 (XBP-1u), shown to be a negative regulator of ATF6, is involved. XBP-lu is thought to play a dual role in the recovery phase of UPR, binding to and degrading both spliced XBP-1 (XBP-1s) and ATF6, slowing UPR-related transcription[6].

\subsection{PERK}

Evolutionarily youngest among transmembrane ER receptors, PERK (PKR-like Endoplasmic Reticulum eIF2 $\alpha$ Kinase) is primarily responsible for translation attenuation contributing to cells' adaptive response to ER stress. Under non-stress conditions in the cell, PERK is also bound to luminal binding protein (BiP)[4]. It is currently theorized that BiP dissociates from PERK and ATF6 before IRE1 [1, 4, $5]$.

The N-terminus of PERK contains an ER luminal stress signal which, upon dissociation from $\mathrm{BiP}$, leads to dimerization and transautophosphorylation of cytosolic protein kinase domains in the PERK dimer. Activated PERK then phosphorylates the alpha subunit of the eukaryotic translation initiation factor-alpha (eIF2 $\alpha$ ) at Serine 51, deactivating it[33].

As eIF $2 \alpha$ is essential for translation initiation, particularly start site recognition, phosphorylated eIF $2 \alpha$ decreases overall levels of protein translation and thus, the rate of new, unfolded proteins entering the ER, thus allowing the ER time to process the existing load of unfolded proteins. However, certain mRNAs, which contain internal ribosome entry site (IRES) sequences, bypass the eIF2 $\alpha$ translation inhibition. One such transcript, ATF4, encodes a cAMP response element-binding transcription factor (C/EBP), facilitating both pro-survival factors, i.e. amino acid transport and synthesis, redox reactions and protein secretion, as well as pro-apoptotic factors, such as expression of transcription factor $\mathrm{C} / \mathrm{EBP}$ ho mologous protein (CHOP) [34].

The PERK pathway is largely regulated by $\mathrm{p} 58$ and $\mathrm{CHOP}$ interactions. p58 expression is induced by ATF6 and inhibits PERK activity through binding to its kinase domain. p58 activity has been shown to take effect a nu mber of hours after PERK activation and is currently thought to serve as a mechanis $m$ for shutting off PERK after a period of activity. Other observed functions of $\mathrm{p} 58$ include co-translational protein degradation and serving as a co-chaperone with BiP. Moreover, PERK is also regulated through a negative feedback loop in which PERK-activated CHOP dephosphorylates eIF $2 \alpha$, removing the block on overall translation[6]. Containing a transcriptional activation domain and a basic-leucine zipper (bZIP) domain, CHOP inhibits Bcl2 expression and increases recognition of ER-stress inducing cells $[1,4,5]$.

It has been shown that PERK is not solely responsible for regulating cyclin D1 accumulation after the activation of the UPR pathway. Hamanaka and colleagues demonstrated that fibroblast cells lacking functional PERK display residual eIF $2 \alpha$ phosphorylation. However, in cells harboring targeted deletions of both PERK and GCN2, another serine/threonine-protein kinase that also phosphorylates eIF2 $\alpha$, the loss of cyclin D1 is attenuated[35]. . This genetic evidence suggests that both PERK and GCN2 cooperatively function to regulate eIF $2 \alpha$ phosphorylation and cyclin D1 translation after UPR activation.

The IRE1 pathway has also been shown to be influenced by the PERK pathway through the activity of microRNA, miR-30c-2* (recently designated miR-30c-2-3p). PERKmediated induction of miR-30c-2* regulates the expression of XBP-1, specifically inhibiting XBP-1-mediated gene transcription and increasing the like lihood that the cell will undergo apoptosis [36].

\section{UPR in Plants}

To date, two distinct UPR signaling pathways have been identified in plants. The first involves proteolytic cleavage of two ER trans membrane transcription factors, bZIP17 and bZIP28, upon translocation to the Golgi system[7, 8], which mechanistically and functionally resembles ATF6-mediated pathway in mammals. The second consists of an unconventional splicing event in bZIP60 by IRE1a and IRE1b, analogous to the splicing of HAC1 by Ire $1 p$ in yeast and XBP- 1 by IRE $1 \alpha$ in mammalian cells.

The Arabidopsis genome encodes two IRE1 homologs, IRE1a (At2g 17520, formerly AtIre1-2) and IRE1b (At5g24360, formerly AtIre 1-1) that share $41 \%$ a mino acid identity. Both members of IRE1 are shown to have largely overlapping expression patterns[3]. However, IRE1b transcript appears to be more abundant in the floral tissue[37].

Recently, a number of reports suggest that IREla and IRE1b may have different physiological roles[10, 38, 39]. IREla appears to play a predominant role in plant immunity, whereas IRE $1 b$ is involved in ab iotic stresses. In Arabidopsis, maize and other monocots, a segment of bZIP60 mRNA has been shown to fold into a structure, which serves as a recognition site for Ire1, consisting of two hairpin loops with three conserved bases in each loop[10, 40]. Recent findings have demonstrated that the unspliced form of bZIP60 contains a transme mbrane doma in (TMD) that, when spliced out, allows the gaining of a nuclear localization signal (NLS), which facilitates translocation into the nucleus to induce the expression of UPR genes [10,40]. Similar to HAC1, wh ich in its unspliced form codes for a weakly active (ten-fold-less potent) transcriptional activator, translation of an unspliced bZIP60 leads to production of a transcription factor that cannot activate expression of ER chaperone genes [41].

Moreover, a protein ho mologous to GCN2, the regulatory protein kinase in yeast, was found in Arabidopsis[42] and termed AtGCN2. AtGCN2 has been shown to play a role in plant starvation responses and is capable of phosphorylating the plant equivalent of eIF $2 \alpha[43,44]$. Additionally, a pathway component reminiscent of ATF4 was recently discovered in Arabidopsis, whereby a transcription factor 
termed TBF1 is controlled on the translational level by two upstream open reading frames (uORFs) in its 5' UTR and its translational de-repression appears to be dependent on eIF $2 \alpha$ phosphorylation[44]. However, unlike in the well characterized dicot Arabidopsis, comparatively little is known about UPR in monocots. The monocot rice, Oryza sativa, has only one known IRE1 homolog, OsIRE1, whose downstream effects are largely unknown. A recent study demonstrates the conservation of the IRE1-bZIP pathway through identification of a protein homologous to Arabidopsis bZIP60, O. sativa protein, OsbZIP74, which exhibits a similar unconventional splicing event in response to ER stress[45].

\section{Conclusions}

In this review, we present the first comprehensive overview of both plant and animal UPR signaling pathways. Across the kingdoms and throughout various levels of life's complexity, the evolution of UPR demonstrates increasing convolution in both form and function. As discussed above, the most conserved structure of UPR is the IRE1, a universal pro-survival sensor and initiator of UPR across species. Other proteins homologous to various components of mammalian UPR's web-like system have been increasingly identified in other organisms suggesting a greater degree of comple xity a mong lo wer eukaryotes than originally thought.

The existence of PERK in mammals and not lower eukaryotes is currently thought to be due to the evolutionary origin of PERK derived from exon shuffling between IRE1 and GCN2[36]. Furthermore, there are currently multiple plausible views on the relationship between PERK and IRE1. It is currently unknown whether the PERK-induced miRNA found to inhibit XBP-1 exp ression represents one of multip le miRNAs, which may exert such an effect. Also of consideration is whether PERK further inhibits IRE1-mediated survival signaling through its actions of specific transcription activation or global translation attenuation[36].

Despite the high level of UPR conservation, variation exists between broad groups of species, as well as similar species such as fungi, supporting previous claims that UPR is intertwined with multiple additional homeostasis regulatory pathways and functions. Given this, basic studies on UPR signal transduction, as well as the relationship between aberrant UPR and disease, constitute very promising areas to offer therapeutical targets. The current limitation of our understanding of the ER stress sensing is the inability to identify small molecules that can enhance or suppress a specific branch of UPR. Future research needs to be dedicated towards that understudied area using highthroughput chemical geno mics approaches.

\section{ACKNOWLEDGEMENTS}

We gratefully acknowledge support from UAB Gulf Oil
Response Pilot Grants to M.S.M. and K.P.M., and the UAB Faculty Development Grant to K.P.M. We also gratefully acknowledge Ms. Cassandra Garbutt for assistance with preparation of the figure.

\section{REFERENCES}

[1] Bernales S, Papa FR, Walter P. Intracellular signaling by the unfolded protein response. Annu Rev Cell Dev Biol, vol. 6, no. 22, pp.487-508, 2000.

[2] Tsai YC, Weissman AM. The Unfolded Protein Response, Degrad ation from Endoplasmic Reticulum and Cancer. Genes Cancer, vol. 1, pp.764-778, 2010.

[3] Noh SJ, Kwon CS, Chung WI. Characterization of two homologs of Irelp, a kinase/endoribonuclease in yeast, in Arabidopsis thaliana. Biochim Biophys Acta, vol.1575, pp.130-134, 2002.

[4] Walter P, Ron D. The unfolded protein response: from stress pathway to homeostatic regulation. Science, vol. 334, pp.1081-1086, 2011.

[5] Ron D, Walter P. Signal integration in the endoplasmic reticulum unfolded protein response. Nat Rev Mol Cell Biol, vol. 8, pp.519-529, 2007.

[6] Mori K. Signalling pathways in the unfolded protein response: development from yeast to mammals. J Biochem, vol. 146, pp. 743-750, 2009.

[7] Liu JX, Srivastava R, Che P, Howell SH. An endoplasmic reticulum stress response in Arabidopsis is mediated by proteolytic processing and nuclear relocation of a membrane-associated transcription factor, bZIP28. The Plant Cell, vol. 19, pp. 4111-4119, 2007.

[8] Liu JX, Srivastava R, Che P, Howell SH. Salt stress responses in Arabidopsis utilize a signal transduction pathway related to endoplasmic reticulum stress signaling. The Plant Journal vol. 51, pp.897-909, 2007.

[9] Liu JX, Srivastava R, Che P, Howell SH. Salt stress signaling in Arabidopsis thaliana involves a membrane-bound transcription factor AtbZIP17 as a signal transducer. Plant signaling \& behavior, vol. 3, pp.56-57, 2008.

[10] Moreno AA, Mukhtar MS, Blanco F, Boatwright JL, Moreno I, Jordan MR, et al. IRE1/bZIP60-mediated unfolded protein response plays distinct roles in plant immunity and abiotic stress responses. PLoS One vol. 2, no. 7, pp.e31944, 2011.

[11] Oikawa D, Tokuda M, Hosoda A, Iwawaki T. Identification of a consensus element recognized and cleaved by IRE1 alpha. Nucleic Acids Research, vol. 38, pp. 6265-6273, 2010.

[12] Zhang K, Kaufman RJ. The unfolded protein response: a stress signaling pathway critical for health and disease. Neurology, vol. 66, pp. S102-109, 2006.

[13] Kimata Y, Kohno K. Endoplasmic reticulum stress-sensing mechanisms in yeast and mammalian cells. Current Opinion in Cell Biology, vol. 23, pp.135-142, 2011.

[14] Pincus D, Chevalier MW, Aragon T, van Anken E, Vidal SE, El-Samad $\mathrm{H}$, et al. BiP binding to the ER-stress sensor Ire1 
tunes the homeostatic behavior of the unfolded protein response. PLoS Biology, vol. 8, pp.e1000415, 2010.

[15] Sidrauski C, Walter P. The transmembrane kinase Irelp is a site-specific endonuclease that initiates mRNA splicing in the unfolded protein response. Cell, vol. 90, pp. 1031-1039, 1997.

[16] Mulder HJ, Nikolaev I. HacA-dependent transcriptional switch releases hacA mRNA from a translational block upon endoplasmic reticulum stress. Eukary ot Cell, vol. 8, pp. 665-675, 2009.

[17] Ogawa N, Mori K. Autoregulation of the HAC1 gene is required for sustained activation of the yeast unfolded protein response. Genes Cells, vol. 9, pp.95-104, 2004.

[18] Feng X, Krishnan K, Richie DL, Aimanianda V, Hartl L, Grahl N, et al. HacA-independent functions of the ER stress sensor IreA synergize with the canonical UPR to influence virulence traits in Aspergillus fumigatus. PLoS Pathogens, vol. 7, pp. e1002330, 2011.

[19] Lee AH, Iwakoshi NN, Glimcher LH. XBP-1 regulates a subset of endoplasmic reticulum resident chaperone genes in the unfolded protein response. Mol Cell Biol, vol. 23, pp.7448-7459, 2003.

[20] Lee K, Tirasophon W, Shen X, Michalak M, Prywes R, Okada $\mathrm{T}$, et al. IRE1-mediated unconventional mRNA splicing and S2P-mediated ATF6 cleavage merge to regulate XBP1 in signaling the unfolded protein response. Genes Dev, vol.16, pp.452-466, 2002

[21] Yoshida H, Matsui T, Hosokawa N, Kaufman RJ, Nagata K, Mori K. A time-dependent phase shift in the mammalian unfolded protein response. Dev Cell, vol. 4, pp. 265-271, 2003.

[22] Yoshida H. Unconventional splicing of XBP-1 mRNA in the unfolded protein response. Antioxid Redox Signal, vol. 9, pp.2323-2333, 2007.

[23] Hetz C. The unfolded protein response: controlling cell fate decisions under ER stress and beyond. Nat Rev Mol Cell Biol, vol.13, pp.89-102, 2012.

[24] Gardner BM, Walter P. Unfolded proteins are Ire1-activating ligands that directly induce the unfolded protein response. Science, vol. 333, pp.1891-1894, 2011.

[25] Luo D, He Y, Zhang H, Yu L, Chen H, Xu Z, et al. AIP1 is critical in transducing IRE1-mediated endoplasmic reticulum stress response. J Biol Chem, vol. 283, pp.11905-11912, 2008.

[26] Hetz C, Bernasconi P, Fisher J, Lee AH, Bassik MC, Antonsson B, et al. Proap optotic BAX and BAK modulate the unfolded protein response by a direct interaction with IRE1alpha. Science, vol. 312, pp. 572-576, 2006.

[27] Gupta S, Deepti A, Deegan S, Lisbona F, Hetz C, Samali A. HSP72 protects cells from ER stress-induced apoptosis via enhancement of IRE1alpha-XBP1 signaling through a physical interaction. PLoS Biol, vol. 8, pp.e1000410, 2010.

[28] Gu F, Nguyen DT, Stuible M, Dube N, Tremblay ML, Chevet E. Protein-tyrosine phosphatase 1B potentiates IRE1 signaling during endoplasmic reticulum stress. J Biol Chem, vol. 279, pp. 49689-49693, 2004.

[29] Haze K, Okada T, Yoshida H, Yanagi H, Yura T, Negishi M, et al. Identification of the G13 (cAMP-response-element-bin ding protein-related protein) gene product related to activating transcription factor 6 as a transcriptional activator of the mammalian unfolded protein response. Biochem J, vol. 355, pp. 19-28, 2001.

[30] Haze K, Yoshida H, Yanagi H, Yura T, Mori K. Mammalian transcription factor ATF6 is synthesized as a transmembrane protein and activated by proteolysis in response to endoplasmic reticulum stress. Mol Biol Cell, vol. 10, pp. 3787-3799, 1999.

[31] Ye J, Rawson RB, Komuro R, Chen X, Dave UP, Prywes R, et al. ER stress induces cleavage of membrane-bound ATF6 by the same proteases that process SREBPs. Mol Cell, vol. 6, pp.1355-1364, 2000.

[32] Yoshida H, Matsui T, Yamamoto A, Okada T, Mori K. XBP1 mRNA is induced by ATF6 and spliced by IRE1 in response to ER stress to produce a highly active transcription factor. Cell, vol. 107, pp. 881-891, 2001.

[33] Hollien J, Weissman JS. Decay of endoplasmic reticulum-localized mRNAs during the unfolded protein response. Science, vol. 313, pp. 104-107, 2006.

[34] Novoa I, Zhang Y, Zeng H, Jungreis R, Hard ing HP, Ron D. Stress-induced gene expression requires programmed recovery from translational repression. EMBO J, vol. 22, pp. 1180-1187, 2003.

[35] Hamanaka RB, Bennett BS, Cullinan SB, Diehl JA. PERK and GCN2 contribute to eIF2alpha phosphory lation and cell cycle arrest after activation of the unfolded protein response pathway. Mol Biol Cell, vol. 16, pp. 5493-5501, 2005.

[36] Byrd AE, Aragon IV, Brewer JW. MicroRNA-30c-2* limits expression of proadaptive factor XBP1 in the unfolded protein response. J Cell Biol, vol. 196, pp. 689-698, 2012.

[37] Koizumi N, Martinez IM, Kimata Y, Kohno K, Sano H, Chrispeels MJ. Molecular characterization of two Arabidopsis Ire1 homologs, endoplasmic reticulum-located transmembrane protein kinases. Plant Physiology, vol. 127, pp. 949-962, 2001.

[38] Nagashima Y, Mishiba K, Suzuki E, Shimada Y, Iwata Y, Koizumi N. Arabidopsis IRE1 catalyses unconventional splicing of bZIP60 mRNA to produce the active transcription factor. Scientific Reports, vol. 1, pp. 29, 2011.

[39] Deng Y, Humbert S, Liu JX, Srivastava R, Rothstein SJ, Howell SH. Heat induces the splicing by IRE1 of a mRNA encoding a transcription factor involved in the unfolded protein response in Arabidopsis. Proc Natl Acad Sci U S A, vol. 108, pp. 7247-7252, 2011.

[40] Li Y, Humbert S, Howell SH. ZmbZIP60 mRNA is spliced in maize in response to ER stress. BMC Res Notes, vol. 5, pp. 144, 2012.

[41] Iwata Y, Koizumi N. An Arabidopsis transcription factor, AtbZIP60, regulates the endoplasmic reticulum stress response in a manner unique to plants. Proc Natl Acad Sci U S A, vol. 102, pp. 5280-5285, 2005.

[42] Zhang Y, Wang Y, Kanyuka K, Parry MA, Powers SJ, Halford NG. GCN2-dependent phosphory lation of eukary otic translation initiation factor-2alpha in Arabidopsis. Journal of Experimental Botany, vol. 59, pp.3131-3141, 2008. 
[43] Lageix S, Lanet E, Pouch-Pelissier MN, Espagnol MC, Robaglia C, Deragon JM, et al. Arabidopsis eIF2alpha kinase GCN2 is essential for growth in stress conditions and is activated by wounding. BMC Plant Biology, vol. 8, no. 8, pp.134, 2008.

[44] Pajerowska-Mukhtar KM, Wang W, Tada Y, Oka N, Tucker CL, Fonseca JP, et al. The HSF-like transcription factor TBF 1 is a major molecular switch for plant growth-to-defense transition. Current Biology, vol. 22, pp.103-112, 2012.

[45] Lu SJ, Yang ZT, Sun L, Sun L, Song ZT, Liu JX. Conservation of IRE1-Regulated bZIP74 mRNA Unconventional Splicing in Rice (Oryza sativa L.) Involved in ER Stress Responses. Molecular Plant, vol. 5, pp.504-514, 2012. 